\title{
State Assignment based on Two-dimensional Placement and Hypercube Mapping
}

\begin{abstract}
This paper addresses the problem of state assignment for Finite State Machines (FSM). This is an important problem in digital system design where added functionality often comes at the expense of a larger (and slower) FSM to control the system. We present a new scheme to solve the graph embedding problem which is the main step in the state assignment process. This new approach places the graph in a two-dimensional array (grid) while minimizing the total edge length, and then maps this two-dimensional array into an $n$-dimensional hypercube with dilation of at most 2 . Experimental results are presented and compared against those of NOVA. These results demonstrate the effectiveness of the proposed approach.
\end{abstract}

Key Words: state assignment, finite state machine, graph embedding, placement, hypercube, dilation. 


\subsection{Introduction}

Controller synthesis is an important problem in the high performance digital system design where added functionality often comes at the expense of a larger (and slower) FSM to control the system. State assignment which takes high-level specifications such as control flowgraphs, state transition tables or state transition graphs as inputs and produces binary codes for the states is an important step in the synthesis of controllers. Once binary codes have been assigned to the states, next-state and output equations are defined and subsequently optimized with classical logic minimization tools. State assignment must therefore be performed in a way that favors simplification of the next-state and output logic (implemented by PLA's, standard cells, ROM's, etc.).

With the rapid advances in circuit complexity and chip density, automatic synthesis tools have become a necessity for integrated circuit design. FSM synthesis techniques must be able to cope with the increasing complexity of the machines with thousands of states [1]. Existing state assignment techniques are however either inefficient or inadequate for handling such large finite state machines. This motivated us to develop a very fast state assignment procedure for handling large machines which is comparable in quality to more sophisticated and elaborate techniques. As PLA's are used extensively in the structured design of high-performance controllers, we will focus on two-level implementation as the target.

\subsection{Previous Work}

Approaches to state assignment can be divided into two broad classes. The first class derives from the classical structure-theory. Examples are [2] and [3] which use algebraic methods based on the partition theory and the reduced dependency criterion. The second class is based on the graphembedding formulation since it formulates the problem as a graph embedding problem on Boolean hypercubes. This class is further divided into two categories. The first category [4][5] formulates the encoding problem as an embedding problem, where an adjacency graph defining adjacency relations between the states is mapped into the hypercube. The second category [6][7] applies symbolic minimization on an unencoded specification followed by extraction of a set of face (input) constraints from the minimized symbolic cover. These constraints are then enforced as much as possible during the embedding.

\subsection{A Unified View}

The concepts of adjacency graph and face constraints are somewhat similar. Both of them describe the desire to assign similar codes to a group of states. The main differences between the two are the followings: (1)The order of logic minimization and encoding is different; (2) One approach involves a cost minimization process while the other involves constraint satisfaction. The first category is different in sense that the goal is to minimize a cost function rather than attempting to satisfy distance relations. The partial constraint satisfaction method described in [8] can be considered as a mixed method.

If we consider the state assignment problem as placing states on an $n$-dimensional hypercube ( $n$ cube), then we can combine the structure-theory-based and graph-embedding-based methods. From the structure theory point of view, each state variable $y_{i}$ introduces a partition $\tau_{i}$ on the set of states, such that two states are in the same block of $\tau_{i}$ if and only if they are assigned the same value of $y_{i}$. Therefore, we can think of each state variable assignment as a hyperplane which cuts 
the $n$-cube. This is similar to a line separating cells in VLSI placement which cuts the chip area into two parts. On the other hand, from the graph-embedding point of view, the set of adjacency relations or input constraints act like the nets or connection constraints in VLSI placement. This viewpoint enables us to combine the structure-theory-based and graph-embedding-based methods into an effective hybrid method.

The graph embedding approach for state assignment is attractive because it can be easily modified to optimize different objective functions. For example, in addition to the minimum area objective mentioned above, it has been used in low power applications [9]. Furthermore, the formulation in [10] that requires some state values to be encoded with non-adjacent binary vectors to improve the testability can be easily modified to adjacency-relations. The state-adjacency graph must be embedded on a Boolean hypercube so that the cost is minimum. Unfortunately, this problem is NP-complete.

In this paper, we propose a very fast yet effective heuristic method for solving this graph embedding problem. The basic idea is to place the adjacency graph in a two-dimensional array (grid) while minimizing the total interconnection length. The placement solution is then mapped into an $n$-dimensional hypercube while nearly preserving the adjacency relations. To obtain good state assignment results, one has to decide what kind of multi-pin net representation should be adopted during the two-dimensional placement, what kind of objective function should be used, and what kind of hypercube mapping should be applied. Some of the contributions of the present paper are in answering these questions either theoretically or empirically. Indeed, we will show that the straight-forward choices are not the right choices for this application. Experimental results of this approach are very good terms of both the CPU time and the circuit area. A preliminary version of this work was published in [11].

The rest of this paper is organized as follows. In section 2 an overview of our proposed approach is given. In section 3 the procedure for constructing the adjacency graph is presented. In section 4 the procedure for two-dimensional placement of the graph is described. In section 5 the procedure for mapping the placement solution into a hypercube of given dimensionality is presented. Experimental results and conclusions are given in section 6 and 7.

\subsection{Outline of the Proposed Approach}

We are given a weighted graph that describes the adjacency between various states or the desirability for giving a group of states similar codes. The larger the weight at an edge, the more desirable it becomes to give the corresponding states adjacent codes. The basic idea is the following. We translate the problem of finding the best hypercube embedding into the following mathematical one. Define the distance, $d_{i j}$, between any two vertices, $i, j$ of an $n$-cube to be the minimal number of edges that must be traversed to get from $i$ to $j$. Then the $n$-cube can be coded so that the $n$-bit codes assigned to vertices $i$ and $j$ differ in only $d_{i j}$ bits. We now wish to assign the nodes of the adjacency graph to the vertices of the $n$-cube so as to minimize the function:

$$
\text { Cost }=\sum_{i, j} w_{i j} \cdot d_{\pi(i), \pi(j)}
$$

where $w_{i j}$ is the weight of edge $(i, j)$ in the adjacency graph, and $d_{\pi(i), \pi(j)}$ is the distance of $\pi(i)$ and $\pi(j)$ where $\pi(i)$ and $\pi(j)$ are coordinates of vertices in the hypercube to which $i$ and $j$ have been 
assigned. Our strategy, code named Hyper-Place, for solving the problem is to find an embedding on a two-dimensional array and then map that solution to a hypercube of minimum dimensionality.

Hyper-Place is composed of three steps as shown in Figure 1.

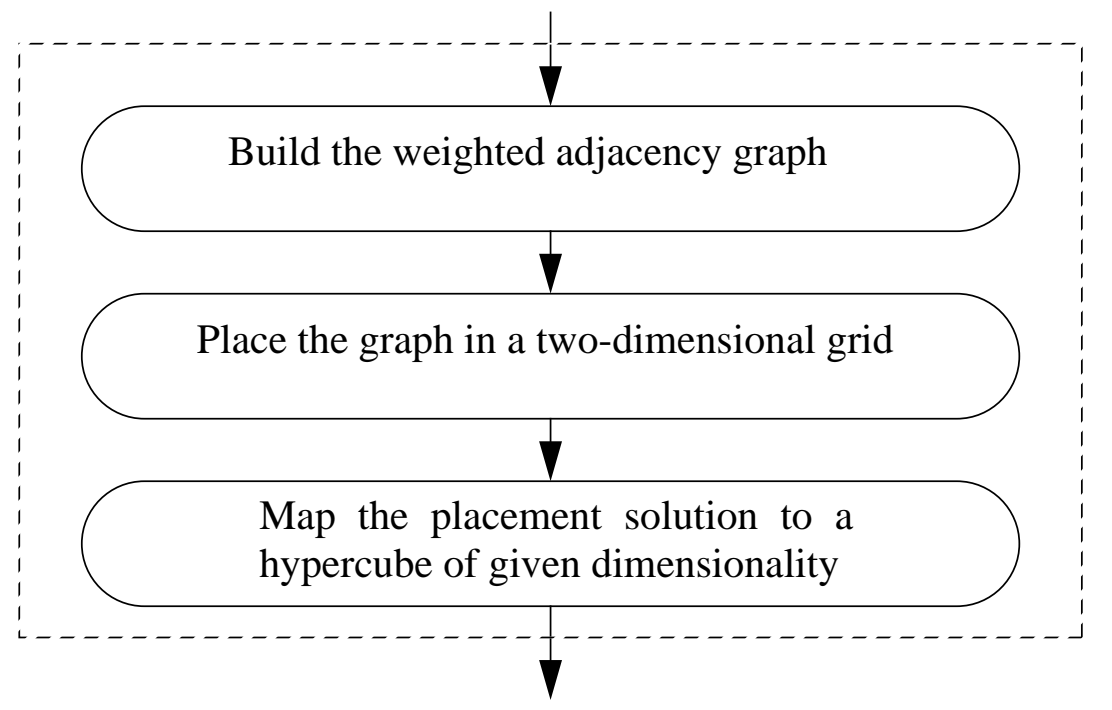

Figure 1: Main procedures in our state assignment approach Hyper-Place

Step 1. An adjacency graph is constructed based on the controller specification. In this graph, each node represents a state and there exists an edge between two nodes if the corresponding states should be given adjacent codes (i.e., codes that differ in only one bit).

Step 2. The adjacency graph is then placed on a two-dimensional array. The placement procedure interleaves a global optimization step with a bi-partitioning step in order to minimize the interconnection length while avoiding congestion on the placement plane.

Step 3. The placement solution is mapped to a hypercube. The question of interest here is how can we find a mapping from the nodes of a two-dimensional placement solution to the nodes of a hypercube so that the relative distances between pairs of nodes in the placement solution is intact after the mapping. A promising result is that: grid neighbors can be always mapped to hypercube nodes such that the worst case distance between grid-neighbors in the hypercube is two [12]. So, as long as we keep vertices adjacent in grids, we can nearly achieve our original goal which was to keep those vertices adjacent in the hypercube.

\subsection{Construction of the Adjacency Graph}

An adjacency graph is formed from high-level FSM specification such as control flowgraphs, state transition tables or state transition graphs. In an adjacency graph, each node represents a state. Between any two nodes, there exists a weighted edge if these two nodes (states) have to be adjacent to reduce the circuit area after state assignment and logic minimization [13].

We adopt the following scheme to generate the adjacency relations. We use symbolic logic mini- 
mization to group together states that are mapped by some input combination into the same nextstate and assert the same output values. It is desirable to give states in each group adjacent codes. This kind of grouping is actually the combination of Type-I and Type-III adjacency heuristics in [13] and is also the state grouping which creates face constraints in [6].

\subsection{Connection Model of Nodes}

There are two ways to describe the connections among nodes representing states in the same state group. The hyperedge model forms one net (hyperedge) connecting all the nodes (states) in each group. That is, the adjacency graph is a hypergraph. The weight of each net is the number of nodes connected by that net. The clique model creates a clique on the nodes belonging to each group. In this case, all connections are two-terminal edges. Modern VLSI placement algorithms tend to use the hyperedge model as it more accurately reflects the connection strengths. In the remainder of this section, we will however show that the clique model is better for the state assignment application.

Definition: An m-subcube of a hypercube $H$ is an $m$-dimensional hypercube contained in $H$.

Definition: The supercube of a graph $G$ embedded on a hypercube $H$, is the smallest $m$-subcube in $H$ which contains $G$.

Definition: The supercube is minimal if $G$ has $n$ nodes and $m=\left\lceil\log _{2} n\right\rceil$.

Theorem 3.1: The minimum edge length embedding of an $N$-node clique on a hypercube $H$ is always contained in a minimal supercube.

Proof. The proof is by induction on $N$. For $N=1$ and $N=2$, the theorem holds. In order to prove the result for arbitrary $N$, we assume that it is true for $N=k$ and the nodes of this clique all locate in an $m$-dimensional hypercube which is also a minimal supercube of the hypercube $H$. We will show that when we add the $(k+1)$ st node to form a new clique with minimum total edge length, that is when $N=k+1$, the result is still correct. Before we continue the proof, we need some lemmas. Proofs of these lemmas are straight-forward and omitted.

Lemma 3.2: The $(k+1)$ st node is adjacent to at least one node of the old clique.

Lemma 3.3: The larger the number of nodes which are adjacent to the $(k+1)$ st node, the shorter the total edge length of the new clique.

We consider two cases.

Case 1) $k=2^{m}$. The $(k+1)$ st node forces an increase in the dimension of its supercube of the new clique which is also minimal.

Case 2) $k<2^{m}$. There is always at least one node within the old supercube which is not occupied. By Lemma 3.3, the minimum edge length embedding will require the new node to be placed inside the old supercube, as in that case the adjacency of the new node can be strictly larger than one. (Compare this with the case where the node goes outside the old supercube in which case the adjacency is at most one.) Clearly, the new supercube will then be the same as the old supercube which is also minimal.

By Theorem 3.1, the group of nodes connected using a clique can be mapped to a minimal supercube. On the other hand, the group of nodes connected by a hyperedge does not necessarily map 
to a minimal supercube. That is, the clique model tends to generate bigger group faces [6] than the hyperedge model. Figure 2 illustrates the concepts described above. Four nodes belonging to a group are placed in a 3-dimensional hypercube. For the hyperedge model, all 3 configurations (Figure 2(a), (b) and (c)) have the same connection cost. Any one of these 3 configurations may be the placement solution which needs minimum total connection length. For the clique model, Figure 2(c) has the minimum total connection length. We note that Figure 2(c) is the configuration that minimizes the final circuit area after logic minimization.

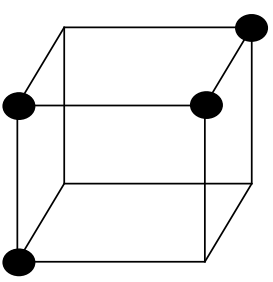

Hyperedge model: Clique model: total length $=3$

total length $=10$

(a)

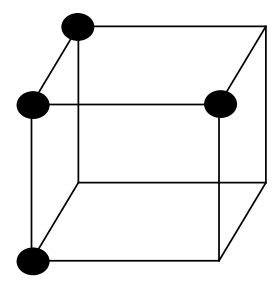

total length $=3$ total length $=9$

(b)

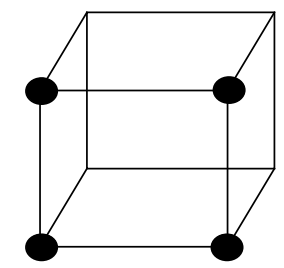

total length $=3$ total length $=8$

(c)

Figure 2: Examples of total connection length using different models

\subsection{Placement on a Two-Dimensional Grid}

To minimize the cost function EQ1, we use a two-phase approach. First, we relax the grid constraints and place the nodes in a continuous plane. Then, this global placement is modified to map the nodes to the grid points [14][15].

\subsection{Global Placement}

During global placement the total edge length among nodes is minimized while neglecting slot constraints. Global placement is interleaved with netlist partitioning. The set of nodes is recursively divided into smaller subsets while the placement area is dissected into subregions. The slicing procedure generates constraints for the next global placement step in subregions. These constraints aim at a better distribution of the nodes over the plane.

\subsubsection{Influence of Various Distance Measures}

The distance function $d_{\pi(i), \pi(j)}$ in EQ1 can be measured in various ways and this in turn affects the final state assignment solution.

Definition: An $l$-norm distance measure is $D\left(x_{i}, x_{j}\right):=\left|x_{i}-x_{j}\right|^{l}$.

For $l=1$, we have the Manhattan distance measure $\left|x_{i}-x_{j}\right|$. For $l=2$, we have the Euclidean square distance measure $\left(x_{i}-x_{j}\right)^{2}$.

We will describe the impacts of a quadratic and a linear objective function on the placement [16] and discuss how that influences our state assignment approach. It is difficult to make definitive statements about which objective function is better in the context of the state assignment problem. Examples are used to demonstrate the impacts of using different objective functions. 
Figure 3 shows a subregion placement situation: two fixed nodes $\mathrm{X}, \mathrm{Z}$ and a movable mode $\mathrm{Y}$. They are connected by nets $a, b, c$ with lengths $l_{a}, l_{b}, l_{c}$, respectively. Minimizing the quadratic objection $\Phi_{q}=l_{a}{ }^{2}+l_{b}{ }^{2}+l_{c}{ }^{2}$ yields the placement in Figure 3(a) with $l_{a}=l_{b}=1 / 2 l_{c}$. Minimizing the linear function $\Phi_{l}=l_{a}+l_{b}+l_{c}$ results in the placement in Figure 3(b) with $l_{a}=l_{b}=0$.

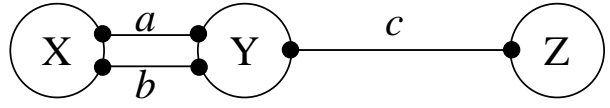

(a) quadratic objective function

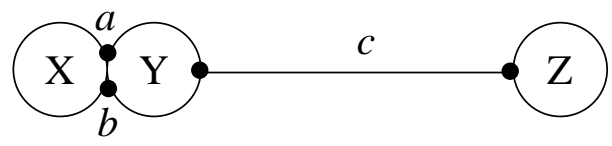

(b) linear objective function

Figure 3: Optimal placement for different objectives

It is generally observed that the quadratic objective function tends to make long nets (net $c$ in Figure 3) shorter, at the expense of increasing the length of the short nets (nets $a$ and $b$ in Figure 3). In other words, the standard deviation of the net lengths is smaller for a quadratic objective function.

Modern VLSI cell placement tools tend to use a linear objective function [16]. This is because a linear objective function directly captures the interconnection length and thus produces denser layout. For example, wire segments $a, b$ in Figure 3(a) may cause more tracks or feedthroughs than zero wire segments $a, b$ in Figure 3(b). However, number of tracks and feedthroughs is not a concern in the state assignment application. On the other hand, the quadratic objective function tends to create a better balance between nodes in relation to their connectivity strengths. For example, in Figure 3(b), if linear objective function is adopted, the ratio of distance of X-Y to Y-Z will be 0 which overstates the adjacency desirability of X-Y to Y-Z. Therefore, a quadratic objective function reflects the actual adjacency demands more accurately than the linear objective function in our application. The statements made here are experimentally confirmed in section 6 .

\subsubsection{Quadratic Programming Formulation and Techniques}

The objective function of the global optimization step is then the weighted sum of the squared rubber band lengths of the edges of the given adjacency graph:

$$
L=1 / 2 \cdot \sum_{v_{i} v_{j}(i \neq j)} c_{i j} \cdot\left(\left(x_{i}-x_{j}\right)^{2}+\left(y_{i}-y_{j}\right)^{2}\right)
$$

where $c_{i j}$ represents the total number of connections between vertex $v_{i}$ and $v_{j} .\left(x_{i}, y_{i}\right)$ and $\left(x_{j}, y_{j}\right)$ represent the locations of $v_{i}$ and $v_{j}$. The cost function can then be rewritten using matrix notation as follows [17]:

$$
L(x, y)=x^{T} B x+y^{T} B y
$$

where $x$ is a vector of the $\mathrm{x}$-coordinates of the vertex locations and $y$ is a vector of the $y$-coordinates. $B$ is a symmetric matrix with $B=D-C$ where $C=\left[c_{i j}\right]$ is the connectivity matrix and $D$ is a 
diagonal matrix with $d_{i i}=\sum_{j=1}^{n} c_{i j}$. It has been shown that if the nodes (vertices) cannot be partitioned into disconnected subsets, then $B$ is positive semi-definite [17]. That means the objective function is a convex function. This fact allows the calculation of a unique global optimum solution and plays an important role in our approach. In addition, $B$ is almost always sparse for practical cases. This enables efficient numerical techniques to be applied to the matrix. Since the coordinate vectors $x$ and $y$ enter separately in the sum of two quadratic forms, we may consider each coordinate independently.

To avoid collapsing all nodes to the center of the placement region, in our implementation, we choose four nodes to be connected to four dummy nodes in the four corners of the placement region. These four dummy nodes will enable us to obtain a non-trivial placement solution. This corresponds to assigning distant codes to these four nodes and thus we pick 4 nodes that are not connected or are weakly connected.

Following the scheme mentioned above, we obtain a global placement as illustrated in Figure 4.

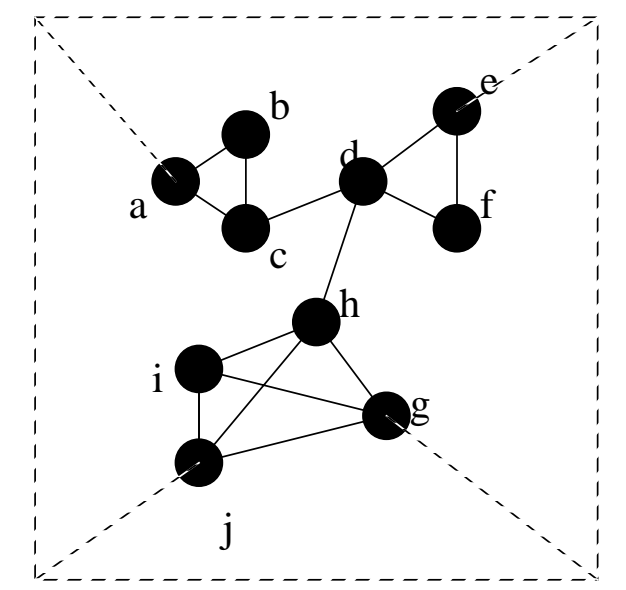

Figure 4: An global placement example

\subsection{Mapping to Grid Positions}

Since the global placement doesn't restrict the nodes to be placed on grid, a detailed placement step has to be performed. The goal of this step is to change the global placement as little as possible while mapping the nodes to the grid positions.

This mapping procedure is done by a minimum squared error linear assignment which maps the movable modules from the global placement to all the legal positions simultaneously. The error to be minimized is $\sum_{i, j=1}^{m} \delta_{i j}\left[\left(x_{i}-m_{j}\right)^{2}+\left(y_{i}-l_{j}\right)^{2}\right]$ where $x_{i}, y_{i}$ are the coordinates of the $i$ th module and $m_{j}, l_{j}$ are the coordinates of the $j$ th legal slot. $\delta_{i j} \in\{0,1\}$ is a selection variable. 
Figure 5 shows the final placement for the solution shown in Figure 4.

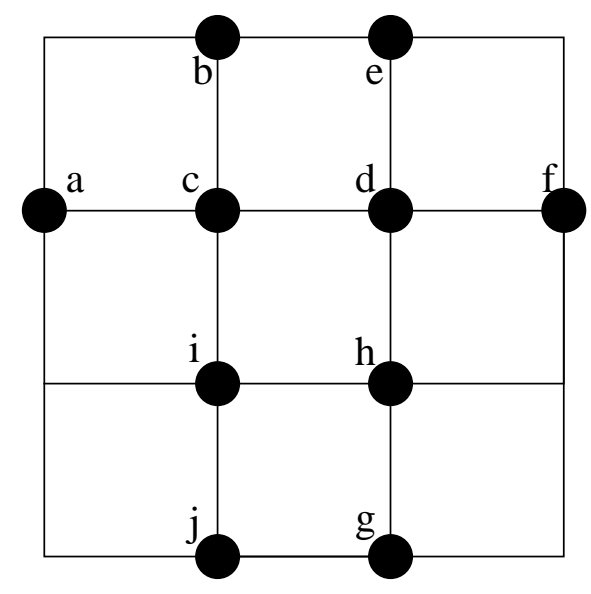

Figure 5: Final Placement

\subsection{Mappings of Grids into Hypercubes}

We want to embed the adjacency graph into hypercube. However, the problem of deciding whether a given graph is embeddable into any dimensioned hypercube is NP-complete [18] and the problem of embedding a given graph into a fixed-sized hypercube is also NP-complete [19]. We thus try to achieve the best partial embedding according to the cost function given by EQ1. Therefore, this section addresses the following graph-mapping problem: given a placement solution on a two-dimensional grid and a hypercube with at least as many nodes as grid points, how can we assign the grid points to hypercube nodes so that the placement cost on the hypercube remains "nearly the same" as that on the grid. In both cases, the costs are calculated by EQ1. The term "nearly the same" will be made more precise later in this section.

In the following discussion, we define the desired properties for the optimal mapping between grids and hypercubes. When these properties are absent we will describe conditions under which those sub-optimal properties can be achieved.

Let $G$ and $H$ denote graph $G$ and hypercube $H$ and $d$ denote a distance function.

Definition: A map $f: G \rightarrow H$ is distance-preserving if $\forall a, b \in G, d(f(a), f(b))=d(a, b)$. We also say that $G$ is a distance-preserving subgraph of $H$.

Definition: A map $f: G \rightarrow H$ is full if a, $\mathrm{b} \in G$ are adjacent exactly if $f(a), f(b) \in H$ are adjacent and vice versa. We also say that $G$ is a full subgraph of $H$.

If we can make a distance-preserving mapping for grids into hypercubes, then we will have the same placement cost in hypercubes as we have in grids.

Theorem 5.1 [20]: If a graph $G$ is a distance-preserving subgraph of some hypercube $H$, then $G$ must be full.

Definition: The minimal hypercube of a two-dimensional array is the smallest hypercube with at least as many nodes as the array. 
Theorem 5.2: A $k$-node two-dimensional array (grid) cannot be a distance-preserving subgraph of its optimal hypercube when $\left\lceil\log _{2} k\right\rceil>4$.

Proof. Suppose $G$ is a $k$-node two-dimensional array and $H$ is an $n$-dimensional hypercube, where $n=\left\lceil\log _{2} k\right\rceil$ and there exists a map $f: G \rightarrow H$. Because $2^{n-1}<k \leq 2^{n}$, we can always find a vertex $v_{x} \in G$ and its corresponding vertex $f\left(v_{x}\right)$ in $H$ where $f\left(v_{x}\right)$ is adjacent to $n$ other vertices which are all mapping nodes of $G$. On the other hand, vertex $v_{x}$ in a two-dimensional array has at most 4 vertices which are adjacent to $v_{x}$. Because $n>4$, we can always find at least one vertex $f\left(v_{y}\right) \in H$ which is adjacent to $f\left(v_{x}\right)$, but $v_{y}$ is not adjacent to $v_{x}$ in $G$. According to Theorem $1, G$ is not a distance-preserving subgraph of hypercube $H$.

By Theorem 5.2, we know that most of the two-dimensional grids are not distance-preserving subgraphs of their optimal hypercubes. Specifically, for those FSM's with more than 16 states, we need to find other mapping properties that could be applied to their sizes of grid.

Definition: The dilation of a grid-hypercube mapping is the maximum distance of grid-neighbors in the hypercube.

We want the dilation of the grid-hypercube mapping be minimum. For example, if dilation 1 mapping can be achieved, then we will have at most the same placement cost in hypercubes as we have in grid. An example of grid-hypercube mapping with dilation 1 is shown in Figure 6. A number of researchers have studied this problem in parallel processing domain, with the following results. Over 61 percent of all two-dimensional grids can be embedded into their minimal hypercubes with a dilation 1 by using binary-reflected Gray codes [21]. Recently, Chan introduced an embedding strategy which makes all the two-dimensional grids to be embeddable in their minimal hypercubes with at most dilation 2 [12].

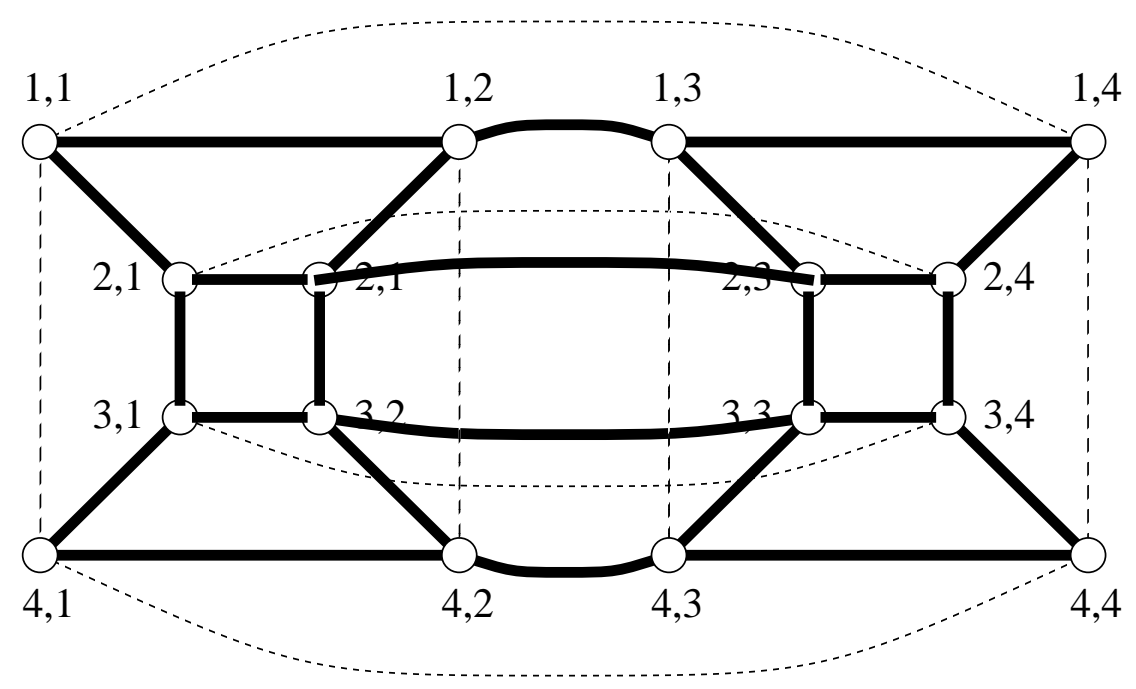

Array edges are shown with solid lines, the unused hypercube edges are shown with dashed lines.

Figure 6: Embedding of a 4x4 grid in a 16-node hypercube

Note that we can always get grid-hypercube mappings with dilation 1 if we use bigger size hypercubes. However, this corresponding to using non-minimal length encoding which is a problem 
that we do not consider in this paper.

Definition: The expansion of grid-hypercube mapping is the ratio of the size (in number of nodes) of the embedding hypercube to the size of the minimal hypercube.

Theorem 5.3 [22]: The smallest hypercube that can embed a $d_{1} \times d_{2} \times \ldots \times d_{k}$ grid using unit dilation has dimension $\left\lceil\log _{2} d_{1}\right\rceil+\left\lceil\log _{2} d_{2}\right\rceil+\ldots+\left\lceil\log _{2} d_{k}\right\rceil$.

As a direct consequence of Theorem 5.3, we know that all two-dimensional grids can be embedded in hypercubes with dilation 1 using an expansion of at most 2. However, the trade-off is that when we use expansion 2 mappings, we need to add one more bit to encode the states. We adopt the approach in [12] which is summarized in Appendix 1.

\subsection{Experimental Results}

To increase the flexibility of this assignment method, we distinguish the nodes into two sets, fixed and movable. Fixed nodes correspond to the states whose codings have been decided in advance. For example, in microprocessors some instructions (states) that invoke co-processors are often assigned fixed codes in advance. Their coordinates could be obtained easily by a reverse transformation of their codes. Also, we choose the ratio of the two dimensions of plane (grid) to be 1. This is based on the observation that the minimum cost of placement solution is often obtained on a square grid. In current implementation, we use GORDIAN [15] to generate the placement solution.

Table 1 shows the statistics of examples tested. These examples include all of the large FSM examples (state number > 20 and product number > 200) in the MCNC logic synthesis and optimization benchmarks [23]. We also generated random examples to cover a more complete range of the size of circuits to be tested. In Table 2, we compare our results with NOVA [7]. In both cases, the code lengths were limited to the minimum number of bits and the number of cubes (product terms) after logic minimization [24] were compared. Basically, NOVA has three modes. The exact mode which produces the best results. However, its CPU run time is very high. For most of these examples, NOVA exact could not produce an answer because the run time (SUN SPARC 1+) was over one week and the process had to be terminated. Therefore, we compare with its default and hybrid modes. In Table 2, area ratio is the ratio of the number of cubes of NOVA to that of our Hyper-Place. CPU time ratio is the ratio of the CPU time (SUN SPARC 1+) of NOVA to that of our Hyper-Place. On average, NOVA default mode produces results with $29 \%$ higher area (number of cubes) and almost the same CPU time compared to Hyper-Place. NOVA hybrid mode takes over 62 times the CPU time required by Hyper-Place and produces almost the same quality of results (in terms of the number of cubes). 
Table 1 Statistics of benchmark examples

\begin{tabular}{|c|c|c|c|c|}
\hline FSM Name & Inputs & Outputs & Products & States \\
\hline s820 & 18 & 19 & 232 & 25 \\
\hline s832 & 18 & 19 & 245 & 25 \\
\hline s1494 & 8 & 19 & 250 & 48 \\
\hline s1488 & 8 & 19 & 251 & 48 \\
\hline x3643 & 3 & 3 & 368 & 64 \\
\hline x4322 & 4 & 2 & 383 & 32 \\
\hline d5326 & 5 & 6 & 384 & 32 \\
\hline d5322 & 5 & 2 & 427 & 32 \\
\hline x4326 & 4 & 6 & 449 & 32 \\
\hline d5324 & 5 & 4 & 511 & 32 \\
\hline d5323 & 5 & 3 & 730 & 32 \\
\hline x5322 & 5 & 2 & 767 & 32 \\
\hline x5321 & 5 & 1 & 809 & 32 \\
\hline x5324 & 5 & 4 & 920 & 32 \\
\hline s298 & 3 & 6 & 1096 & 218 \\
\hline x5643 & 5 & 3 & 1464 & 64 \\
\hline x5642 & 5 & 2 & 1535 & 64 \\
\hline tbk & 6 & 3 & 1569 & 32 \\
\hline x5641 & 5 & 1 & 1617 & 64 \\
\hline x63210 & 6 & 10 & 1649 & 32 \\
\hline x6326 & 6 & 6 & 1793 & 32 \\
\hline m6325 & 6 & 5 & 2048 & 32 \\
\hline
\end{tabular}


Table 2 Comparisons of NOVA and our Hyper-Place

\begin{tabular}{|c|c|c|c|c|c|c|c|c|c|c|}
\hline \multirow[b]{2}{*}{ Example } & \multicolumn{4}{|c|}{ NOVA(default) } & \multicolumn{4}{|c|}{ NOVA(hybrid) } & \multicolumn{2}{|c|}{ Hyper-Place } \\
\hline & cubes & $\begin{array}{l}\text { area } \\
\text { ratio }\end{array}$ & $\begin{array}{c}\mathrm{CPU} \\
\text { time(s) }\end{array}$ & $\begin{array}{l}\mathrm{CPU} \\
\text { time } \\
\text { ratio }\end{array}$ & cubes & $\begin{array}{l}\text { area } \\
\text { ratio }\end{array}$ & $\begin{array}{c}\mathrm{CPU} \\
\text { time(s) }\end{array}$ & $\begin{array}{l}\mathrm{CPU} \\
\text { time } \\
\text { ratio }\end{array}$ & cubes & $\begin{array}{c}\mathrm{CPU} \\
\text { time(s) }\end{array}$ \\
\hline s820 & 85 & 1.13 & 1.3 & 0.24 & 76 & 1.01 & 4.2 & 0.78 & 75 & 5.4 \\
\hline s832 & 71 & 0.97 & 1.3 & 0.25 & 72 & 0.99 & 4.2 & 0.79 & 73 & 5.3 \\
\hline s1494 & 149 & 1.14 & 3.1 & 0.09 & 139 & 1.06 & 388.8 & 10.83 & 131 & 35.9 \\
\hline s1488 & 141 & 1.07 & 2.7 & 0.07 & 133 & 1.01 & 416.6 & 11.23 & 132 & 37.1 \\
\hline x3643 & 271 & 1.04 & 7.8 & 0.17 & 251 & 0.97 & 2560.2 & 55.90 & 260 & 45.8 \\
\hline$x 4322$ & 261 & 1.30 & 2.7 & 0.18 & 155 & 0.77 & 732.6 & 50.18 & 201 & 14.6 \\
\hline d5326 & 295 & 1.15 & 19.2 & 1.14 & 233 & 0.91 & 3621.9 & 215.60 & 257 & 16.8 \\
\hline d5322 & 217 & 1.10 & 5.5 & 0.28 & 233 & 1.18 & 984.4 & 50.74 & 197 & 19.4 \\
\hline $\mathrm{x} 4326$ & 359 & 1.24 & 29.7 & 1.62 & 266 & 0.92 & 3322.0 & 12.08 & 290 & 18.3 \\
\hline d5324 & 374 & 1.34 & 26.1 & 1.43 & 248 & 0.89 & 2148.0 & 118.02 & 279 & 18.2 \\
\hline d5323 & 423 & 1.16 & 59.7 & 3.21 & 330 & 0.90 & 3212.3 & 172.70 & 365 & 18.6 \\
\hline x5322 & 428 & 1.33 & 28.3 & 1.46 & 232 & 0.72 & 1718.4 & 88.58 & 323 & 19.4 \\
\hline $\mathrm{x} 5321$ & 525 & 1.24 & 7.1 & 0.53 & 378 & 0.89 & 2301.0 & 171.72 & 423 & 13.4 \\
\hline x5324 & 557 & 1.37 & 8.9 & 0.49 & 388 & 0.95 & 2284.8 & 125.54 & 408 & 18.2 \\
\hline s298 & 723 & 1.07 & 58.1 & 0.65 & 624 & 0.92 & 5460.1 & 60.67 & 678 & 90.0 \\
\hline x 5643 & 750 & 1.48 & 75.1 & 0.65 & 656 & 1.09 & 4020.3 & 34.84 & 518 & 115.4 \\
\hline x5642 & 840 & 2.20 & 129.9 & 0.95 & 347 & 0.91 & 3070.7 & 22.50 & 382 & 136.5 \\
\hline tbk & 176 & 1.76 & 17.1 & 0.85 & 154 & 1.54 & 922.9 & 45.92 & 100 & 20.1 \\
\hline x5641 & 936 & 1.37 & 28.4 & 0.33 & 833 & 1.22 & 3889.7 & 45.12 & 682 & 86.2 \\
\hline x63210 & 1072 & 1.23 & 21.0 & 2.66 & 859 & 0.99 & 988.4 & 125.11 & 872 & 7.9 \\
\hline x6326 & 421 & 1.49 & 23.2 & 1.27 & 278 & 0.98 & 388.9 & 21.25 & 283 & 18.3 \\
\hline m6325 & 1675 & 1.19 & 228.1 & 11.46 & 1341 & 0.95 & 6619.5 & 332.64 & 1406 & 19.9 \\
\hline Total & & 1.29 & & 1.005 & & 0.99 & & 62.84 & & \\
\hline
\end{tabular}


Table 3 compares the results of state assignment using different objective functions in the placement phase. The approach using the linear objective function costs $6 \%$ more cubes than the one using the quadratic objective function which confirms our observation in Section 4.1.1.

Table 3 Comparisons of using linear and quadratic objective functions

\begin{tabular}{|c|c|c|c|}
\hline \multirow{2}{*}{ Example } & \multicolumn{2}{|c|}{ Linear obj. fun. } & \multirow{2}{*}{$\begin{array}{l}\text { Quadratic } \\
\text { obj. fun. } \\
\text { cubes }\end{array}$} \\
\hline & cubes & area ratio & \\
\hline s820 & 80 & 1.07 & 75 \\
\hline s832 & 68 & 0.93 & 73 \\
\hline s1494 & 139 & 1.06 & 131 \\
\hline s1488 & 133 & 1.01 & 132 \\
\hline x3643 & 253 & 0.97 & 260 \\
\hline x4322 & 184 & 0.92 & 201 \\
\hline d5326 & 264 & 1.03 & 257 \\
\hline d5322 & 188 & 0.95 & 197 \\
\hline x4326 & 293 & 1.01 & 290 \\
\hline d5324 & 278 & 1.00 & 279 \\
\hline d5323 & 370 & 0.99 & 365 \\
\hline x5322 & 310 & 0.96 & 323 \\
\hline x5321 & 477 & 1.13 & 423 \\
\hline x5324 & 402 & 0.99 & 408 \\
\hline s298 & 713 & 1.05 & 678 \\
\hline x5643 & 593 & 1.14 & 518 \\
\hline x5642 & 388 & 1.02 & 382 \\
\hline tbk & 188 & 1.88 & 100 \\
\hline x5641 & 777 & 1.14 & 682 \\
\hline x63210 & 935 & 1.07 & 872 \\
\hline x6326 & 280 & 0.99 & 283 \\
\hline m6325 & 1534 & 1.09 & 1406 \\
\hline Total & & 1.06 & \\
\hline
\end{tabular}




\subsection{Conclusions}

In this paper, we presented a new state assignment approach Hyper-Place which runs as fast as the NOVA's default mode but produces same quality results as the NOVA's hybrid mode. This was made possible by breaking the hypercube embedding problem into two steps: (1) mapping of the adjacency graph to a grid; (2) mapping the solution on the grid to one on a minimum dimensionality hypercube with dilation at most two. Hyper-Place is thus able to handle large FSM's (up to 500 states, equations with more than 1000 product terms) efficiently and robustly. 


\section{Appendix 1}

\section{Grid-hypercube mapping procedure}

Notation: Let $N_{k}$ denote the sequence of $k$-bit binary-reflected Gray code, and let $N_{k}(p)$ denote the $(p+1)$ st element in the sequence $N_{k}$. For example, $N_{1} \equiv(0,1), N_{2} \equiv(00,01,11,10), N_{3} \equiv$ $(000,001,011,010,110,111,101,100)$ and $N_{3}(4) \equiv 110$.

Assume all logs are in base 2. Suppose we are given an $x$ x $y$ grid $G$.

CASE 1. $y>2^{\lfloor\log x\rfloor+\lfloor\log y\rfloor+1} \quad$ or $\quad x=2^{\lfloor\log x\rfloor} \quad$ or $\quad y=2^{\lfloor\log y}$.

Then, embed $\mathrm{G}$ into its optimal hypercube using the binary-reflected Gray code strategy, and hence, with dilation 1 .

CASE 2. otherwise.

Assume, without loss of generality, $x \leq \frac{3}{2} 2^{\lfloor\log x\rfloor}$ (otherwise, G could be rotated by 90 degrees).

Since $x y \leq 2^{\lfloor\log x\rfloor+\lfloor\log y\rfloor+1}$, our objective is to label each node of the grid with a unique $(\lfloor\log x\rfloor+\lfloor\log y\rfloor+1)$-bit binary number with the restriction that grid-neighbors can only differ in at most 2 bit positions.

Step 1. Determine the first $\lfloor\log x\rfloor$ bits of each node's label.

Create $2^{\lfloor\log \rfloor\rfloor}$ “chains", each of which is described by a $y$-vector of 1 's and 2 's.

The vector of the first chain is

$$
\left(a_{1,1}, a_{1,2}, \ldots, a_{1, y}\right)=\left(\left\lceil\frac{x}{2^{\lfloor\log x\rfloor}}\right\rceil,\left\lceil\frac{2 x}{2^{\lfloor\log x\rfloor}}\right\rceil-\left\lceil\frac{x}{\left.2^{\lfloor\log x}\right\rfloor}\right\rceil, \ldots,\left\lceil\frac{y x}{2^{\lfloor\log x\rfloor}}\right\rceil-\left\lceil\frac{(y-1) x}{2^{\lfloor\log x\rfloor}}\right\rceil\right)
$$

The vector for the $i$ th chain $\left(i=2,3, \ldots, 2^{\lfloor\log x\rfloor}\right)$ is

$$
\left(a_{i, 1}, a_{i, 2}, \ldots, a_{i, y}\right)=\left(\left\lceil\frac{(i-1) x}{2^{\lfloor\log x\rfloor}}\right\rceil-\left\lceil\frac{(i-2) x}{2^{\lfloor\log x\rfloor}}\right\rceil, a_{i-1,1}, a_{i-1,2}, \ldots, a_{i-1, y-1}\right)
$$

Each chain vector represents a "chain". Figure 7 is an example.

Chain vector: $(2,1,2,1,1,2,1,1,2,1,2)$

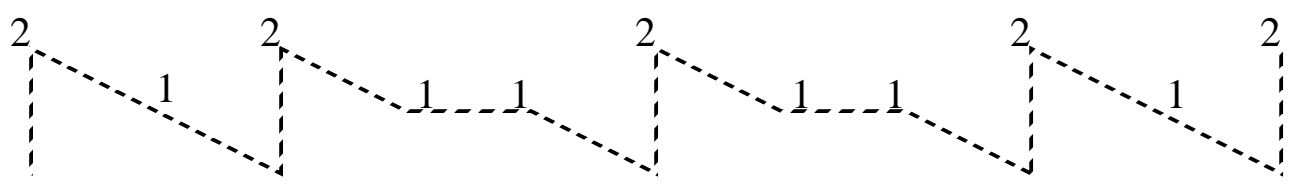

Figure 7: An example of chain vector and "chain" 
An $11 \times 11$ grid is associated with

$(2,1,2,1,1,2,1,1,2,1,2)$

$(1,2,1,2,1,1,2,1,1,2,1)$

$(1,1,2,1,2,1,1,2,1,1,2)$

$(2,1,1,2,1,2,1,1,2,1,1)$

$(1,2,1,1,2,1,2,1,1,2,1)$

$(1,1,2,1,1,2,1,2,1,1,2)$

$(2,1,1,2,1,1,2,1,2,1,1)$

$(1,2,1,1,2,1,1,2,1,2,1)$

and depicted in Figure 8.

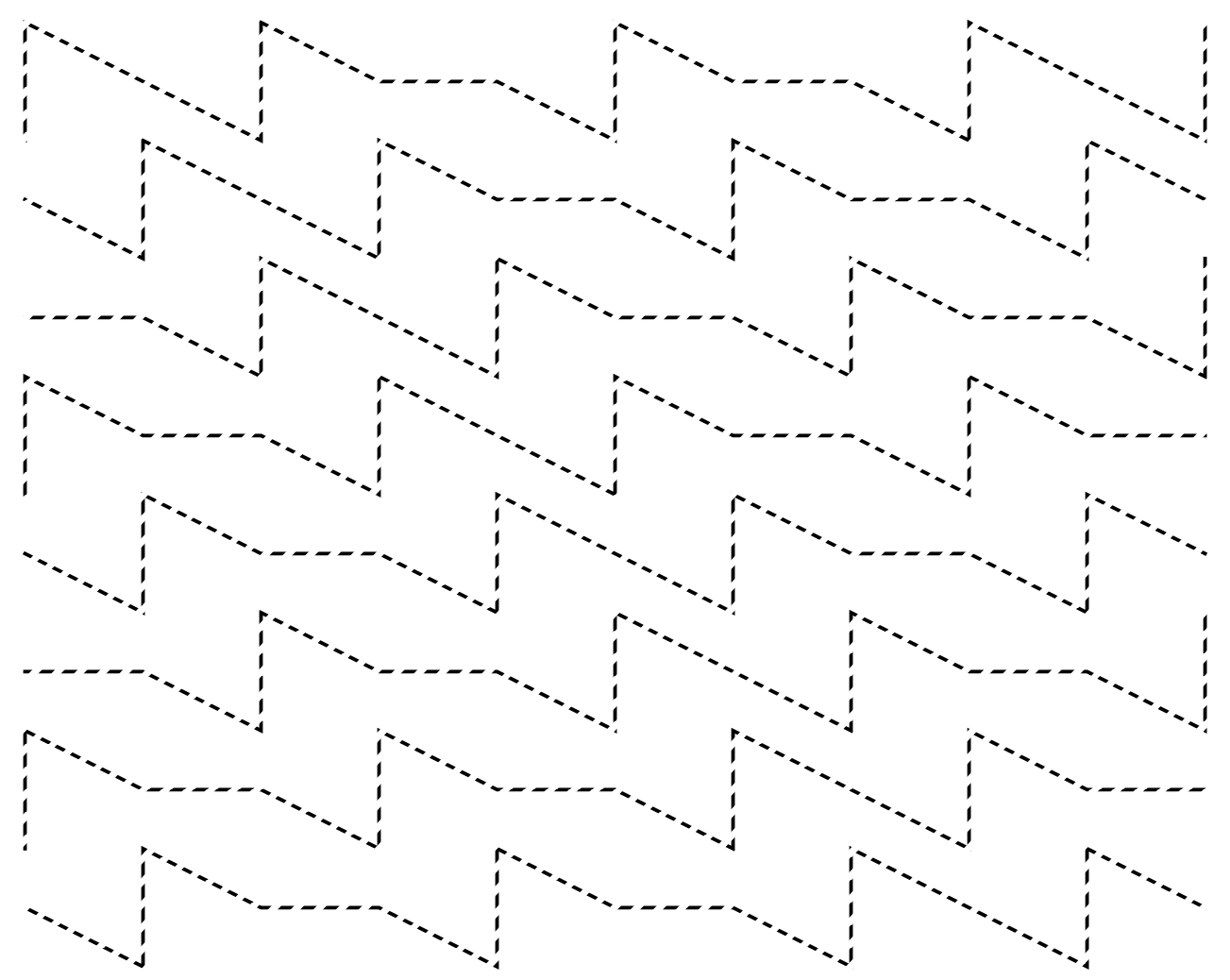

Figure 8: Chains for $11 \times 11$ grid

Aligning the nodes of the graph in Figure 8 into 11 rows, or in general $x$ rows, we have graph $G_{1}$ in Figure 9. The chains will cover the $x \times y$ grid completely (proof can be found in [12]).

Then, each node of the $x \times y$ grid belonging to the $i$ th chain is given $N_{\lfloor\log x\rfloor}(i-1)$ as the first $\lfloor\log x\rfloor$ bits of its $(\lfloor\log x\rfloor+\lfloor\log y\rfloor+1)$-bit label.

Step 2. Determine the first $\lfloor\log y\rfloor+1$ bits of each node's label.

First marking: The $j$ th node of the $i$ th chain is marked with $\left(t_{i}+j\right) \bmod 2^{\lfloor\log y\rfloor+1}$, where $t_{1}=-1$ and 


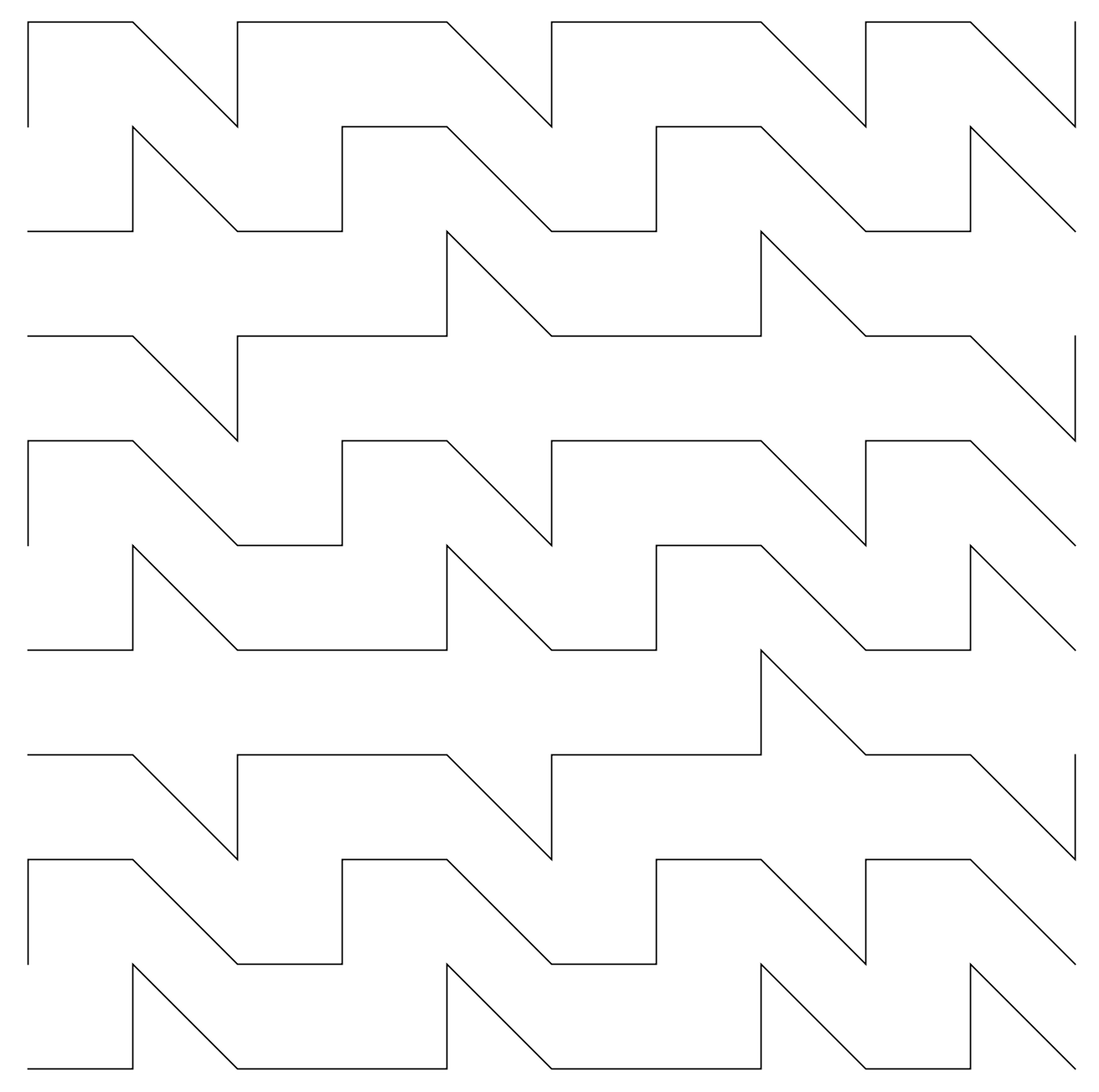

Figure 9: Redrawn chains: $G_{1}$

$t_{i}=t_{i-1} a_{i, 1}+1$. After this marking, we have the graph in Figure 10 for the $11 \times 11$ grid. Note that, the marks of adjacent nodes of the grid differ by at most 2 (in mod $\left.2^{\lfloor\log y\rfloor+1}\right)$.

Second marking: Each mark $t$ is changed to $\lfloor t / 2\rfloor$. Note that, marks for adjacency nodes in the grid will differ by at most 1 (in mod $2^{\lfloor\log y\rfloor}$ ). Then, the chains are horizontally extended to have exactly $2^{\lfloor\log y\rfloor+1}$ nodes each. We get Figure 11 and call it the marked graph $G_{2}$.

Next, we color each node of the grid either red or black so that

(a) two nodes marked with the same number belonging to the same chain are colored differently, and

(b) two adjacent nodes marked with different numbers belonging to different chains are colored the same.

The reason we can accomplish this coloring is shown in [12]. However, condition (a) ensures that 


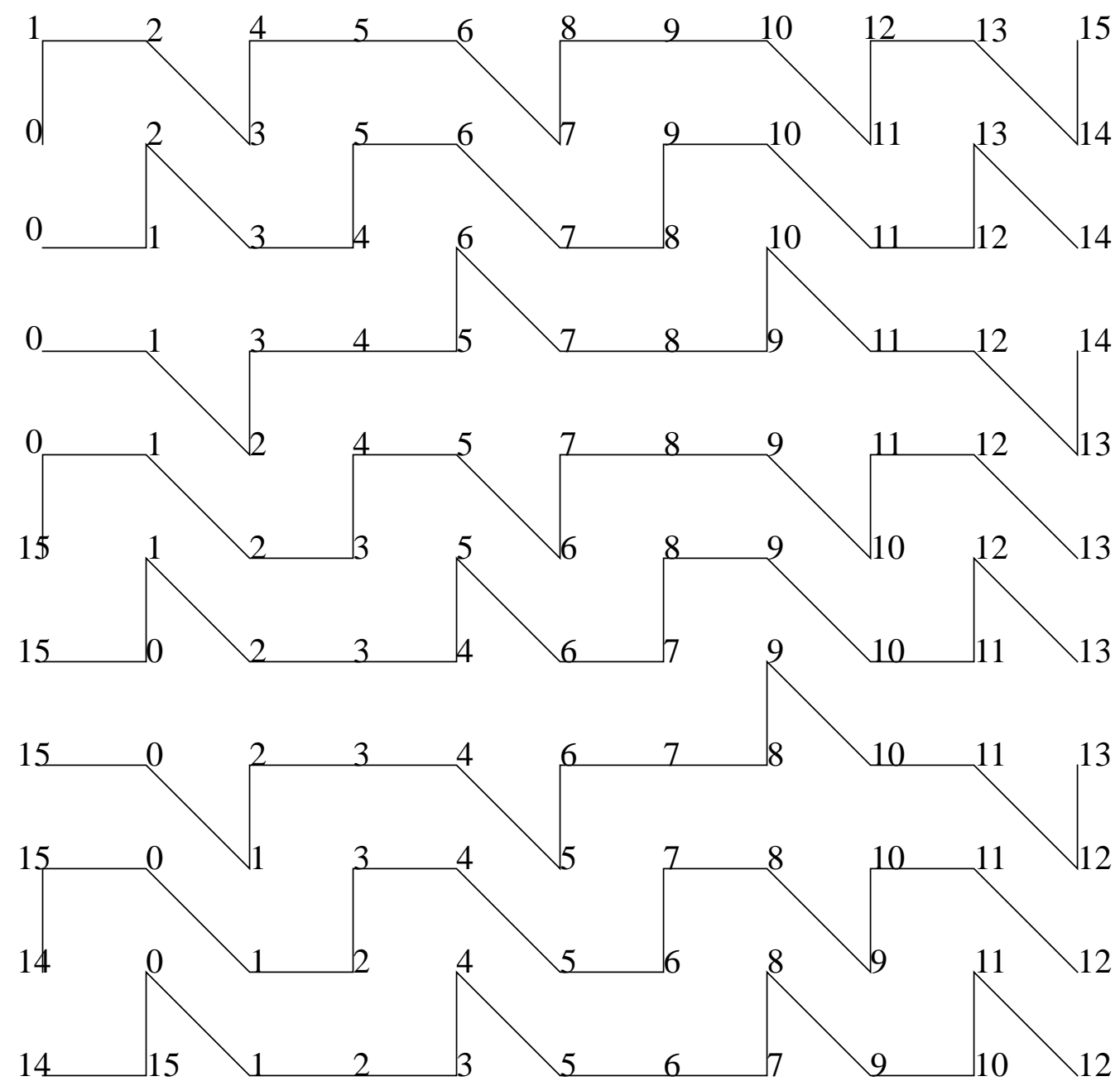

Figure 10: First marking

each node of the grid is indeed mapped to a unique node in the hypercube, and condition (b) ensures that dilation 2 is achieved for adjacent nodes of different chains.

Finally, with such a coloring, a red node marked $t$ is given $0 N_{\lfloor\log y}(t)$ as the last $\lfloor\log y\rfloor+1$ bits of its label, while a black node marked $t$ is given $1 N_{\lfloor\log y\rfloor}(t)$ as its the last $\lfloor\log y\rfloor+1$ bits. In this way adjacent nodes of the same chain will differ in at most 2 bits position of their last $\lfloor\log y\rfloor+1$ bits and share the same initial $\lfloor\log x\rfloor$ bits, making a dilation of 2; adjacent nodes of different chains differ in at most 1 bit position of their last $\lfloor\log y\rfloor+1$ bits and 1 bit position of their initial $\lfloor\log x\rfloor$ bits, again making for a dilation of 2 . 


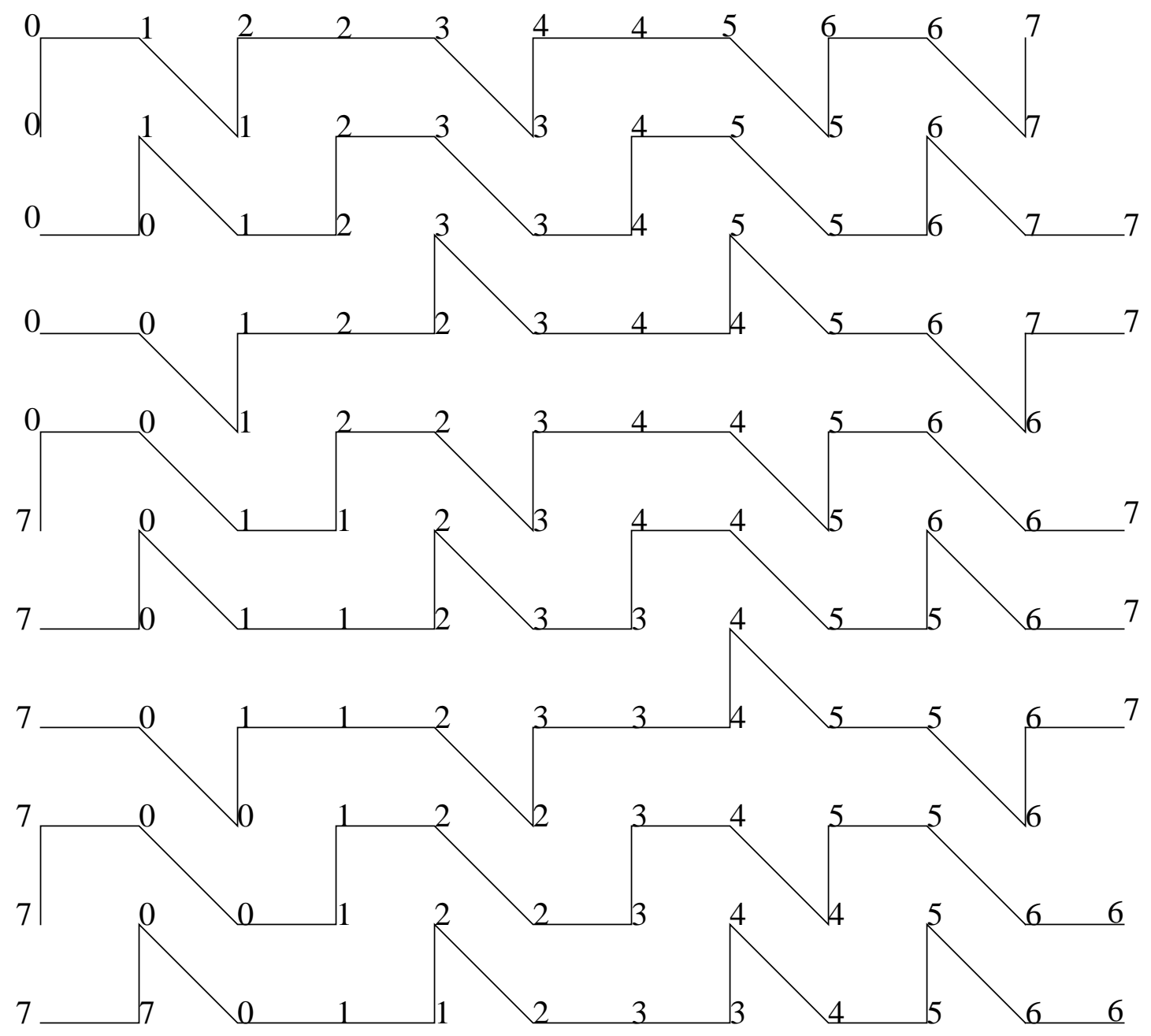

Figure 11: Second marking: $G_{2}$ 


\section{References}

[1] C. Duff and G. Saucier, "State assignment based on the reduced dependency theory and recent experimental results," pp. 222-225, 1991.

[2] R. E. Stearns and J. Hartmanis, "On the state assignment problem for sequential machines II,” IRE Tâns. Elect. Comput., vol. EC-10, pp.593-603, 1961.

[3] T. A. Dolotta and E. G. McCluskey, "The coding of internal states of sequential machines," IEEE Trans. Elect. Comput., vol. EC-13, pp.549-562, 1964.

[4] D.B. Armstrong, "A programmed algorithm for assigning internal codes to sequential machines," IRE Tians. Elect. Comp. , vol. EC-11, pp. 466-472, 1962.

[5] S. Devadas, H.T. Ma, A. R. Newton and A. L. Sangiovanni Vincentelli, "MUSTANG: state assignment of finite state machines for multi-level logic implementations," IEEE Tans. on CAD, pp. 1290-1300, 1988.

[6] G. De Micheli, R. K. Brayton and A. L. Sangiovanni Vincentelli, "Optimal state assignment for finite state machines," IEEE Tians. on CAD, pp.269-284, 1986.

[7] T. Villa and A. L. Sangiovanni-Vincentelli, "NOVA: State Assignment of Finite State Machines for Optimal Two-Level Logic Implementations," IEEE Tans. on CAD, pp. 905924, 1990.

[8] S. Devadas, A. R. Wang, A. R. Newton and A. L. Sangiovanni Vincentelli, "Boolean decomposition in multilevel logic optimization," IEEE Journal of Solid-State Circuits, vol. 24, pp. 399-407, 1989.

[9] K. Roy and S. Prasad, "SYCLOP: synthesis of CMOS logic for low power applications," IEEE International Conference on Computer Design, pp.464-467, 1992.

[10] S. Devadas, H.T. Ma, A. R. Newton and A. L. Sangiovanni Vincentelli, "A synthesis and optimization for fully and easily testable sequential machines," IEEE Tians. on CAD, pp. 1100-1107, 1989.

[11] Shihming Liu, Massoud Pedram and Alvin M. Despain, "A fast state assignment procedure for large FSMs," 32nd Design Automation Conference Proceedings, pp.327-332, 1995.

[12] M. Y. Chan, "Dilation-2 embeddings of grids into hypercubes," Proceedings of the 1988 International conference on Parallel Processing, pp. 295-298, 1988.

[13] D. Lewin, Computer-aided design of digital systems, Crane/Russak, 1977.

[14] Ren-Song Tsay, Ernest Kuh and Chi-Ping Hsu, "Proud: a sea-of-gates placement algorithm,” IEEE Design \& Tst of Computers, pp.44-56, Dec. 1988.

[15] J. M. Kleinhans, G. Sigl and F. M. Johannes, "GORDIAN: A new global optimization/rect- 
angle dissection method for cell placement," ICCAD-88, pp. 506-509, 1988.

[16] G. Sigl, K. Doll and F. M. Johannes, "Analytical placement: a linear or quadratic objective function?” 28th DAC, pp.427-432, 1991.

[17] K. M. Hall, “An r-Dimensional Quadratic Placement Algorithm,” Management Science, vol. 17, pp.219-229, 1970.

[18] D.W. Krumme, N. Venkataraman and G. Cybenko, "Hypercube embedding is NP-complete," Proc. Hypercube Conf., SIAM, 1985.

[19] G. Cybenko, D.W. Krumme and N. Venkataraman, "Fixed hypercube embedding," Information Processing Letters, v.25, pp.35-39, 1987.

[20] D. Z. Djokovic, "Distance-preserving subgraphs of hypercubes," J. Combinatorial Theory (B), pp.263-267, 1973.

[21] Y. Saad and M. H. Schultz, “Topological properties of hypercubes," Res. Report 389, Dept. of Computer Science, Yale Univ. 1985.

[22] J. E. Brandenburg and D. S. Scott, "Embeddings of communication trees and grids into hypercubes, Intel Scientific Computers Report \#280182-001, 1985.

[23] Saeyang Yang, "Logic synthesis and optimization benchmarks user guide," Ersion 3.0, MCNC, 1991.

[24] R. K. Brayton, G. D. Hatchtel, C. T. McMullen and A. L. Sangiovanni Vincentelli, "Logic Minimization Algorithms for VLSI Synthesis,” Kluwer Academic, 1984. 